\title{
Economía, política y conflictividad minera durante las presidencias de Evo Morales en Bolivia (2006-2016)
}

\section{Alejandro Schneider*}

\section{RESUMEN}

El presente artículo intenta esbozar algunas cuestiones sobre las relaciones que se establecieron entre la clase trabajadora minera y los gobiernos de Evo Morales Ayma en Bolivia (2006-2016).

En ese sentido, en un primer momento, este ensayo busca exponer las principales características que tuvo la política económica del Movimiento Al Socialismo (MAS) en esos primeros diez ańos de mandato presidencial. En una segunda parte, el artículo aborda algunas problemáticas referidas a los conflictos gremiales suscitados entre el proletariado minero y las medidas implementadas por el gobernante indígena. En particular, se observan un conjunto de protestas que se dieron alrededor de una serie de yacimientos mineros así como también sobre un cúmulo de temas alrededor de su relación con los asalariados del sector y con los empresarios cooperativistas en sus dos primeras gestiones presidenciales.

Además, como parte central de este análisis, se indagará sobre las distintas prácticas sindicales y políticas que fue adoptando la Central Obrera Boliviana (COB) en esta última década; en particular, su cambiante relación con el presidente.

Palabras clave: Bolivia, Evo Morales, política económica, conflictividad y minería.

\section{Abstract}

This present article attempts to outline some questions about the relations established between the working class mining and governments of Evo Morales Ayma in Bolivia (2006-2016).

In that sense, at first, this essay seeks to expose the main characteristics that had the economic policy of Movement Al Socialismo (MAS) in the first ten years of presidential mandate. In the second part, the article addresses some issues relating to labor conflicts arising between the mineworkers and the measures implemented by the indigenous ruler. In particular, a series of protests that occurred around a series of mineral deposits as well as on a range of issues around their relationship with employees in the sector and cooperative entrepreneurs in his first two presidential administrations are observed.

In addition, as a central part of this analysis, we inquire about the various unions and political practices that was taking the Central Obrera Boliviana $(\mathrm{COB})$ in the last decade; in particular its changing relationship with the president.

Keywords: Bolivia, Evo Morales, economic policy, conflict and mining.

*aschneider98@yahoo.com.ar 


\section{INTRODUCCIÓN}

partir del año 1985, emergió una nueva Bolivia. El país del altiplano que nació con la Revolución de 1952 comenzó un proceso de modificaciones estructurales. El ascenso al poder del líder histórico del Movimiento Nacionalista Revolucionario (MNR) Víctor Paz Estensoro marcó el cierre de un ciclo en el que él fue un protagonista destacado. La piedra angular que dio inicio a estas transformaciones fue la aplicación del Decreto Supremo 21.060; desde entonces se entró en una época signada por una fuerte ofensiva neoliberal sobre la sociedad.

Tras proclamar que "Bolivia se nos muere", Paz inauguró un drástico programa de medidas económicas que acarreó un nuevo acuerdo de dominación sobre la sociedad civil. Se buscó recrear y restablecer la autoridad estatal perdida en los últimos años, en particular, sobre la clase obrera y el campesinado. En ese escenario, el mundo de la minería expresó los cambios más radicales. La crisis del mercado internacional del estaño y el constante quebranto de la productividad en las minas fueron los argumentos que se emplearon para iniciar el desmantelamiento progresivo de las empresas productoras.

El núcleo central de las reformas estructurales impulsadas por el Palacio Quemado fue el desmantelamiento de la poderosa Corporación Minera de Bolivia (COMIBOL). En el plazo de un año se cerraron más de cincuenta minas estatales. La citada empresa despidió a más de las dos terceras partes de sus obreros, incrementando las tasas de desocupación y subempleo, convirtiendo en pueblos fantasmas a numerosos distritos erigidos alrededor del trabajo del subsuelo. A la par de este fenómeno, como parte del mismo modelo económico implementado, fue creciendo el sector informal a través del comercio al menudeo y de los servicios. En otras palabras, desde entonces se fue configurando un mundo laboral distinto al producido por la Revolución de 1952.

A partir de 1985 se inició la debacle de la hasta entonces principal organización obrera del país: la Federación Sindical de Trabajadores Mineros Bolivianos (FSTMB). Este fenómeno fue acompañado no sólo por el aumento del desempleo, sino también por una disper- 
sión (eufemísticamente llamada relocalización) del proletariado del subsuelo a través de todo el territorio. Sin embargo, si bien su presencia militante disminuyó, el ciclo de rebeliones abierto a partir de las guerras del agua (2000) y del gas (2003) junto con la llegada al gobierno del Movimiento al Socialismo (MAS) lo volvió a instalar en la escena política.

El ascenso a la presidencia de Evo Morales Ayma, en enero de 2006, representó un momento de esperanza para extensos sectores de la población. Aunque el gobierno logró una cierta redistribución de la riqueza, tras varios años de intensas disputas regionales y política, la situación económica y social de la mayoría de la ciudadanía no se modificó en sus aspectos sustanciales. A pesar de ello, en los comicios de 2009 y de 2014, una gran parte de la población renovó en ambas oportunidades la confianza en el MAS.

En un escenario de permanente transformación, el presente artículo busca sintetizar los principales lineamientos económicos de estos diez años de gobierno, para luego detenerse en el análisis de una serie de tensiones sociales y políticas en torno a la relación que la administración de Morales mantuvo con los trabajadores mineros. En particular, se observan un conjunto de conflictos y enfrentamientos que se dieron alrededor de una serie de yacimientos mineros así como también sobre un cúmulo de temas y problemas alrededor de su relación con los asalariados y con los cooperativistas en sus dos primeras gestiones presidenciales.

\section{La política EXTRACTIVISTA DEL MAS}

Desde los primeros días de su gestión, en enero de 2006, Morales y su vicepresidente, Álvaro García Linera, anunciaron la intención de conducir a Bolivia hacia un modelo de "socialismo comunitario". Uno de los pilares centrales para alcanzar ese objetivo era el de tratar, previamente, de lograr la plena soberanía económica. Estas ideas fueron reiteradas en numerosas ocasiones; por ejemplo, cuando se promulgó la nueva Constitución Política del Estado en febrero de 2009.

Dentro de ese mismo marco conceptual, García Linera sostuvo que el Estado Plurinacional de Bolivia se encuentra transitando ha- 
cia el "socialismo comunitario" aunque reconoció que este proceso de transición hacia el socialismo puede durar "años, décadas y hasta siglos"; mientras tanto su logro dependerá de consolidar los seis ejes de lucha que se propone el gobierno: "superar el capitalismo, suprimir la explotación capitalista, detener la destrucción de la naturaleza y la vida, superar la división social en clases sociales, terminar con el racismo, y la contienda por el bienestar y la hermandad universal" ("La Prensa", La Paz, febrero 8 de 2010).

Sin embargo, a diez años de haberse iniciado la gestión presidencial del MAS, la realidad que experimenta el país es bien diferente a la que publicitan sus apologistas e ideólogos oficiales. Lejos de esas aspiraciones, en términos globales, las administraciones del líder cocalero se caracterizaron por continuar con el sistema primario exportador que identifica al territorio desde la llegada de los españoles. La base económica permanece atada a una lógica extractivista nutrida por los altos precios mundiales de algunas materias primas que vende al mercado mundial. De ese modo, se ha ido consolidando una economía capitalista dependiente, subdesarrollada, apoyada en el saqueo de los recursos naturales y la sobrexplotación de la fuerza de trabajo, donde el endeudamiento externo constituye una pieza central del engranaje de su funcionamiento. En ese sentido, bajo sus gobiernos, se incrementaron los compromisos financieros con el exterior. Según el último informe del Banco Central Boliviano, los principales organismos acreedores multilaterales son la Corporación Andina de Fomento (CAF), el Banco Interamericano de Desarrollo (BID), el Banco Mundial (BM), junto con China, Brasil, Alemania y Corea del Sur ("El Deber", Santa Cruz de la Sierra, mayo 11 de 2016).

Como producto de esta orientación económica, Bolivia alcanzó una tasa promedio de 5,1\% de crecimiento del Producto Interno Bruto (PIB) entre 2006 y 2014, a pesar de la crisis mundial del 2009 (Ministerio de Planificación del Desarrollo, en adelante MPD, 2016: 48). Estas cifras, en gran medida, se explican por el significativo incremento de las exportaciones de materias primas dentro de un ciclo de alza en los precios internacionales de los commodities. Es necesario considerar que las exportaciones en el 
2005 llegaron a los 2.867 millones de dólares, para alcanzar en 2014 a 12.856 millones (Instituto Nacional de Estadística, en adelante INE, 2014).

La producción primaria de bienes no renovables siguió teniendo un peso central en la estructura económica, tal como lo explicita el Plan de Desarrollo Económico y Social 2016 - 2020. Las exportación de productos primarios provenientes de las ramas de hidrocarburos y minería se han convertido en los ejes nodales que explican los logros obtenidos en los citados indicadores macroeconómicos. Según el INE (2014), ambas actividades representaron el 66,9\% de las exportaciones: hidrocarburos $(51,2 \%)$ y minerales $(15,8 \%)$; por su parte, las operaciones vinculadas al agro y a la industria manufacturera intervinieron tan sólo con el 33,1\% de las exportaciones (MPD, 2016: 24). Por su parte, bajo las presidencias del MAS las ventas al exterior de textiles, prendas de vestir, joyería de oro, muebles de madera, etc., cayeron abruptamente frente a la participación dominante de los envíos de gas natural y minerales.

En correspondencia con ello, el gobierno durante mucho tiempo se vanaglorió de sostener una balanza comercial con superávit; sin embargo, este panorama empezó a cambiar desde hace un año. A partir del primer trimestre de 2015 se ha comenzado a registrar una caída abrupta, provocando un saldo deficitario. De acuerdo con el Instituto Boliviano de Comercio Exterior (IBCE) esto se produjo debido al derrumbe del precio internacional de los hidrocarburos (sobre todo el petróleo) y los minerales. Según datos oficiales, el monto de las exportaciones alcanzó en julio de 2015 la cifra de 5.449 millones de dólares, frente a 7.867 millones de similar período en el 2014, lo que representó una disminución de 2.418 millones. Mientras que el valor de las importaciones se situó en 5.479 millones de dólares, frente a 5.763 millones de julio del 2014, mostrando un descenso de 258 millones. Con escasas excepciones, se generalizó el descenso de las ventas a los principales socios comerciales: con Brasil disminuyeron en 35\%, con Argentina bajaron en 38\%, con Estados Unidos en un $44 \%$ y con Colombia se redujeron un $23 \%$. Esta tendencia continuó durante el presente año: para febrero de 
2016, Bolivia volvió a registrar un saldo deficitario en su balanza comercial. De acuerdo con los organismos gubernamentales, el déficit se incrementó de 14,3 millones a 229,4 millones de dólares, según datos comparativos entre el primer bimestre de 2015 y el de $2016^{1}$.

Este modelo de balanza comercial es ilustrativo del funcionamiento de los países primarios exportadores. Explicita también el comportamiento inflexible que adoptan las importaciones (por la debilidad del aparato productivo y la escasa o nula industrialización), lo que ratifica la condición volátil y dependiente que tienen estas estructuras económicas que responden sin intermediación a los ciclos de precios y los volúmenes del mercado mundial.

Por su parte, la situación en la cuenta corriente de la balanza de pagos reflejó un superávit continuo (en promedio 6\% del PIB, entre 2006-2014). En íntima vinculación con estas cifras, el gobierno logró que en esos años, las Reservas Internacionales Netas (RIN) obtuvieran un incremento notable: en términos del PIB, 46,2\% en 2014, situándose entre las más altas del mundo (MPD, 2016: 49). Sin embargo, este panorama comenzó a cambiar a partir del 2015. En parte, según el Banco Central de Bolivia, se produjo una declinación debido a la disminución de los ingresos provenientes por la exportación de Yacimientos Petrolíferos Fiscales Bolivianos (YPFB), como consecuencia de la reducción del precio del crudo y sus derivados en el mercado internacional. Además, por el incremento de las transferencias de fondos al exterior en dólares por parte de las entidades financieras para atender las operaciones de importación de sus clientes ("Página Siete”, La Paz, mayo 31 de 2016).

Todo este esquema descansa en la prioridad otorgada a la explotación de bienes primarios, como ya se señaló. Esto se expresa tanto en la inversión privada como en las políticas públicas dirigidas hacia las ramas extractivas. Asimismo, se manifiesta en la deformación estructural de la economía que otorga una importancia central al incremento de la productividad en los sectores vinculados a la exportación en detrimento del ámbito doméstico. De acuerdo con el Ministerio de Hidrocarburos y Energía (2011) el principal destino de la producción de hidrocarburos durante este período se dirigió 
hacia los mercados externos, con cerca de un $70 \%$; en contraste con su empleo en los procesos de refinación y transformación en combustibles en las refinerías del país donde alcanzó al 16\% y al consumo como combustible — gas natural domiciliario e industrial- que alcanzó a un $14 \%$.

Asimismo, esto se encuentra en íntima correspondencia con los diversos programas de infraestructura, por medio de su financiamiento externo, que facilitan la libre circulación de mercancías hacia el mercado mundial. Según diferentes informes que realiza la Comisión Económica para América Latina y el Caribe (CEPAL), la mayor parte de la Inversión Extranjera Directa (IED) en Latinoamérica se orientó a la esfera de los recursos naturales, en menor medida al sector de servicios y de las manufacturas, evidenciando una manifiesta tendencia a primarizar las economías regionales. Bolivia no es la excepción: entre el 2005 y el 2012, la IED ha pasado de 459 millones de dólares a 2032 millones, siendo su principal destino la extracción de hidrocarburos con $76,2 \%$ del total de las inversiones, lo que equivale a 1550 millones de dólares (Rivasplata, 2015). En ese contexto se comprende que el interés de los capitales provenientes de la CAF, el BM, el BID, de China y de Brasil estén dirigidos a proyectos de transporte, hidroeléctricas y a plantas de procesamientos de bienes primarios.

Un caso que ejemplifica esta situación es el fuerte vínculo que se ha establecido con el gigante asiático a partir de la visita de Evo a Pekín en el año 2011. Desde ese momento, en el marco de lo que el gobierno consideró a esta relación comercial como una "asociación estratégica”, se suscribieron dieciséis acuerdos en materia de comunicaciones, seguridad alimenticia, energía, minería y transporte. De esa manera, las firmas chinas se convirtieron en las mayores contratistas de estas inversiones, beneficiándose con numerosas licitaciones y adjudicaciones directas. Además, todo esto se encuadra dentro del nuevo esquema de contrataciones públicas: Bolivia cede la estimación de los presupuestos y el posible costo de las inversiones a las compañías ejecutoras de los proyectos, desistiendo el Estado su papel de regulador del capital. 
La presencia de empresas de ese origen se encuentran en distintos rubros: Camce Engineering Co. maneja un tramo de la obra de ferrovía Bulo Bulo-Montero, la planta de sales de potasio en Uyuni, la ejecución (junto con capitales mexicanos) de la hidroeléctrica Misicuni, etc.; la firma Sinohydro Corporation Limited interviene en la ruta Ichilo-Ivirgarzama y en la construcción de sus puentes, en el proyecto vial Padilla-El Salto, en la Planta hidroeléctrica San José, etc.; la empresa Harzone Industry Corp. construye los puentes Beni II y Madre de Dios; la firma Sinopec International Petroleum construye el puente San Buenaventura, el oleoducto Camiri-Santa Cruz, entre otros emprendimientos ("El Deber", Santa Cruz de la Sierra, marzo 3 de 2016)².

La presencia de estas empresas asiáticas han recibido distintos tipos de críticas. Por un lado, algunas firmas chinas que trabajan en Bolivia tienen a su cargo obras estatales que están fuera de su especialidad o del área principal de su negocio; por el otro, varias de estas compañías, como China Camce Engineering y Sinohydro, enfrentaron cuestionamientos ya sea por el incumplimiento de los tiempos en los contratos firmados, por aspectos técnicos, por factibilidad económica y por problemas tanto en el ámbito ambiental como laboral. Por ejemplo, en el caso de esta última compañía, se la ha acusado de incumplir en la legislación obrera; así, el Centro de Estudios para el Desarrollo Laboral y Agrario (CEDLA) observa que esta corporación incurrió en la no suscripción de contratos de trabajo, la falta de pago por horas extras, la carencia de seguro de salud, la adopción de insuficientes medidas de seguridad ocupacional, en descuentos arbitrarios de salarios, despidos injustificados, acoso laboral y el desconocimiento del fuero sindical (CEDLA, 2016).

En otro orden de temas, cabe subrayar que según el IBCE, para el año 2015, Bolivia exportó treinta y seis productos a China, siendo los nueve principales de origen mineral; en cambio, llegaron desde el país asiático 3.868 productos, entre otros, celulares, motocicletas, neumáticos, computadoras, equipos electrónicos, piezas de helicópteros y aviones (IBCE, 2015).

Como hemos indicado, China no fue el único país beneficiado en los intercambios comerciales. Brasil, desde el año 2006, también 
ha desempeñado un papel de primer orden en las inversiones en la construcción de infraestructura vial para conectar diversas regiones de Bolivia con los centros mundiales. En ese marco, una de las empresas que ha participado en estos rubros fue la Constructora OAS Ltda. El vínculo comercial y financiero con esta firma se concentró fundamentalmente en la ejecución de proyectos de la Red Vial Fundamental, bajo responsabilidad de la Administradora Boliviana de Carreteras. Entre esa fecha y el 2009, el gobierno suscribió con esa compañía tres contratos por alrededor de ochocientos millones de dólares. Cabe indicar que la Contraloría General del Estado, realizó diversas observaciones a todos esos acuerdos; sin duda, la más resonante de todas esas críticas fue la ocasionada con el proyecto carretero que pretendió unir Villa Tunari (Cochabamba) con San Ignacio de Moxos (Beni), atravesando el Territorio Indígena Parque Nacional Isiboro Sécure (TIPNIS) (Rivasplata, 2015).

En todo este esquema de funcionamiento, la administración de Morales en forma permanente enfatiza que el éxito de su modelo económico se debe a la nacionalización de los hidrocarburos. Ahora bien, al respecto conviene subrayar algunas cuestiones. En primer lugar, la nacionalización se hizo en el marco de consolidar tanto el modelo productivo extraccionista como el de gestar una épica propagandista del gobierno alrededor de su discurso "socialista". En ese sentido, la medida fue dictada el primero de mayo de 2006, día internacional de los trabajadores, a través del decreto "Héroes del Chaco", en referencia a los bolivianos que fallecieron en la guerra contra el Paraguay (1932 -1935). En segunda instancia, a diferencia de otras estatizaciones, esta disposición no implicó la expulsión o el enfrentamiento con las empresas privadas del sector sino más bien su permanencia bajo nuevas condiciones impositivas, sin impugnar su presencia hegemónica. En esa coyuntura, la norma legal se orientó hacia un incremento del gravamen cobrado a las multinacionales beneficiadas por el ciclo alcista de los precios del petróleo, aunque sin incidir tanto en el manejo real de la extracción como en la cantidad de utilidades y ganancias que las firmas giran a sus respectivas casas matrices en el exterior. De ese modo, la ley apuntó tan sólo a reformar el régimen tributario con el 
fin de mejorar la percepción fiscal. En ese esquema, cabe observar que no se dio un auténtico proceso de incautación porque la fase central del proceso de producción lo siguieron controlando las empresas extranjeras. De acuerdo con un informe del CEDLA (2016), las firmas internacionales continuaron siendo las mayores responsables de la producción de gas natural y de hidrocarburos líquidos en contraste con la participación de YPFB; de ahí se comprende por qué ninguna compañía privada se retiró de este negocio.

En otras palabras, la consecuencia inmediata de la denominada nacionalización de los hidrocarburos se expresó en la notable recaudación impositiva, la cual le permitió al gobierno aplicar tanto una política de ampliación del consumo doméstico como de mayor gasto estatal. Todo esto en un marco en el que se acentuó la escasa diversificación del aparato productivo agudizando el modelo extractivista primario exportador. Asimismo, esta situación acarrea una serie de consecuencias estrechamente ligadas: primero, reafirma una dependencia respecto a los recursos minerales no reemplazables; segundo, genera una alta vulnerabilidad de la economía frente a las fluctuaciones del mercado internacional.

\section{LA PRODUCCIÓN AGRÍCOLA PARA LA EXPORTACIÓN}

$\mathrm{Al}$ igual que en la exportación de minerales e hidrocarburos, las presidencias del MAS han favorecido la producción de materias primas agrícolas a fin de satisfacer la demanda del mercado externo antes que las necesidades de la población. Una muestra de esta orientación política han sido las modificaciones parciales que propició el mandatario indígena sobre la reforma agraria y la ley que regula el Instituto Nacional de Reforma Agraria (INRA). En ese sentido, el gobierno privilegió la defensa de la propiedad de la tierra siempre que esta cumpla con una función económica-social; según esa interpretación, se incluyen dentro de las normas legales vigentes todas aquellas grandes propiedades (incluso las explotadas por las empresas privadas) que declaren sus propósitos productivos. De ese modo, la administración de Morales, además de garantizar el respeto al gran capital privado, concedió inversiones en infraestructura productiva y transporte, precios rentables 
y la permanencia del tipo de cambio monetario, entre otros favores. Asimismo, durante todos estos ańos, subsidió y mantuvo inalterablemente a un precio bajo el valor del diesel como combustible para el sector rural. En esa coyuntura, al calor del incremento mundial de los precios de algunos productos agrícolas (como la soya, el girasol, la caña de azúcar), el agro-negocio se extendió como forma de desarrollo de la producción agropecuaria en sintonía con una mayor extranjerización de la propiedad, en particular, en el departamento de Santa Cruz donde creció la presencia de empresarios agrícolas ganaderos de Brasil y Argentina (Urioste, 2011).

Por otro lado, todo esta política económica de apoyo al proceso de expansión de la frontera agrícola no siempre ha resultado exitosa. Uno de los casos más impactantes de este fracaso fue la movilización y la resistencia de los pueblos indígenas del TIPNIS ${ }^{3}$. A lo largo de toda su gestión, Morales atravesó serios conflictos con dicho sector. La principal forma en que se manifestó esta pugna fue a través de las marchas opositoras a La Paz en contra de la construcción del proyecto carretero que pretendió pasar por ese territorio intangible. Si bien la idea de edificar la autopista era un proyecto de larga data, el presidente en el año 2010 firmó un crédito con el Banco Nacional de Desarrollo Económico y Social de Brasil para financiar esa inversión. Cabe observar que detrás de este proyecto se encuentran tanto intereses sectoriales de capitales nativos y extranjeros involucrados en la explotación agropecuaria para la exportación, como grupos cultivadores de coca de la región del Chapare (Quiroga et al., 2012).

En ese sentido, las demandas de la Confederación Indígena del Oriente Boliviano (CIDOB) no sólo buscaron detener la construcción de la carretera y de frenar a los cocaleros sino que también incorporaron numerosos reclamos, entre otros: la titulación colectiva de tierras y territorios, la gestión territorial indígena autónoma, el uso y aprovechamiento exclusivo de los recursos naturales renovables existentes en su territorio, la consulta a través de sus instituciones y la creación de una universidad indígena.

En esta coyuntura, la movilización más importante fue la VIII Marcha de los Pueblos Indígenas por la Defensa del Territorio, la 
Autonomía Indígena y los Derechos de los Pueblos Indígenas efectuada entre los meses de agosto y octubre de 2011. La marcha tuvo un amplio respaldo de la población, sobre todo, tras la violenta represión del gobierno con heridos ejercida en la localidad de Chaparina. Tras esos acontecimientos, luego de recorrer cerca de seiscientos cincuenta kilómetros, la llegada a La Paz fue recibida calurosamente por distintos sectores de la ciudadanía que se volcaron a las calles para expresar su apoyo a los indígenas (Fundación UNIR, 2011 y Fortún, 2012).

Frente a ello, el primer mandatario promulgó la Ley $\mathrm{N}^{\circ} 180 \mathrm{de}$ intangibilidad del TIPNIS. Sin embargo, la concesión fue momentánea; poco tiempo después Evo impulsó la realización de una nueva movilización solicitando la anulación de la anterior norma. Ésta estuvo encabezada por el Consejo Indígena del Sur (CONISUR), entidad creada para sostener los proyectos presidenciales en la región. Como corolario, tras haber arribado a La Paz en enero de 2012, se estableció la Ley $\mathrm{N}^{\circ} 222$ que explicitó la convocatoria a una consulta previa para la construcción de la carretera. Ante ello, los originarios de la CIDOB efectuaron una IX marcha en el mes de junio de ese año, a la vez que decidieron rechazar el cuestionario desde las propias comunidades del TIPNIS.

De manera clara y contundente en las protestas de los pueblos indígenas de las tierras bajas salió a la luz una de las principales tensiones inherentes en el denominado "Proceso de Cambio" del gobierno: la lógica que privilegia el desarrollo de un capitalismo industrial a partir de la integración al mercado mundial frente a otra que sostiene la defensa de determinados valores ecológicos, sociales y comunitarios. El enfrentamiento por el control del TIPNIS se convirtió en un hecho emblemático para la presidencia de Morales debido a que diversas leyes nacionales reconocen su doble condición: como parque nacional y como territorio indígena. Además, la Constitución del 2009 establece para los pueblos indígenas la preexistencia de sus naciones, su autogobierno, el manejo de los recursos naturales y el derecho a la consulta. En consecuencia, el bloque social que hasta entonces se hallaba representado en el Pacto de Unidad, 
entró en crisis: dos de las cinco centrales campesinas que apoyaban al gobierno rompieron con éste, mientras que hubo otros sectores, como los cocaleros de Cochabamba y la Confederación Sindical de Comunidades Interculturales de Bolivia, que exigieron la construcción de la carretera ${ }^{4}$.

\section{UNA LIMITADA INDUSTRIALIZACIÓN ACORDE AL MODELO ECONÓMICO} Como parte de esta orientación política, Bolivia continúa teniendo una economía escasamente industrializada; su desarrollo no ha superado desde hace muchas décadas el 18\% del PIB; a su vez, la misma se concentró en la elaboración de alimentos, bebidas y tabaco (Arce 2016: 21). Para las presidencias del MAS, el pensamiento y la práctica industrializadora sólo se encuentra en función de mejorar (ańadir un poco de valor agregado) los productos primarios que se venden en el mercado internacional. Así, la manufactura que se efectúa sobre algunos bienes se hace en torno a la fundición de algunos minerales como el estańo, bismuto, antimonio y cobre, y la refinación de oro y plata, los cuales (en gran medida) son para la exportación. Por ende, no se han desarrollado proyectos industriales que generen máquinas que elaboren bienes de capital para dejar de depender de insumos y materias primas importadas (Arce, 2014).

Por otro lado, cabe subrayar que algunos emprendimientos industriales comenzaron a efectuarse tras una serie de violentos enfrentamientos, como fueron los casos de la planta procesadora de cítricos de Caranavi (La Paz) y la Planta de Fundición de Karachipampa (Potosí) (Schneider, 2014).

En ese sentido, por fuera de estos sectores mencionados, la producción industrial se ha concentrado en la rama de alimentos y bebidas, la cual se caracteriza por su escasa (o más bien nula) planificación manufacturera, su insuficiente generación de empleo, su minúscula inversión de capital y su tenue aporte al conjunto del PBI. Así, por ejemplo, el rubro textil se encuentra conformado por pequeñas empresas que emplean, en promedio, veinticinco trabajadores, y micro empresas, con diez empleados ("Página Siete", La Paz, abril 27 de 2014). 
En términos generales, para los años 2005-2013, se evidenció una pronunciada caída en la participación de la Industria Manufacturera: los textiles y prendas de vestir sufrieron un descenso del $21 \%$ y las otras producciones -verbigracia, las industrias de maquinaria, metálicos básicos y diversos- declinaron en 22\% (Arce, 2014).

De acuerdo con la gestión fiscal del año 2014, las utilidades de las industrias más grandes (que representan el $80 \%$ del sector) cayeron $8,7 \%$. Según Hugo Siles Espada, economista de la Cámara Nacional de Industrias, entre los principales motivos que intervinieron para la declinación de los beneficios se encuentra la extraordinaria competencia de los bienes manufacturados importados (en la que incluye también a los provenientes del contrabando), el incremento del "costo laboral" y al tipo de cambio fijo de la moneda frente la devaluación de las monedas en los países vecinos ("Página Siete", La Paz, noviembre 16 de 2015).

En los últimos años, uno de los sectores donde claramente se manifiesta esta grave situación fue en la manufactura textil. Esto ha generado el cierre de varios establecimientos con su correspondiente ola de despidos; en gran medida, esto ha ocurrido tanto por la importación masiva de vestimenta de procedencia china como porque se han cerrado las ventas al mercado norteamericano. En cuanto al primer factor, en el quinquenio 2008-2013, el ingreso proveniente del gigante asiático se duplicó. En el año 2013, el valor de las compras ascendió a 18,3 millones de dólares frente a los \$US 9,7 millones registrados en 2009 , lo que equivale a un crecimiento del $89 \%$. Los volúmenes también se incrementaron en $90 \%$, al pasar de 12.200 toneladas a 23.167, según datos oficiales ("La Razón”, La Paz, junio 22 de 2014). En segundo término, la crisis en el sector se debió por la cancelación de los beneficios que otorgaba la Ley de Preferencias Arancelarias Andinas y Erradicación de la Droga en el 2008; en este caso, la medida dispuesta por el gobierno de Estados Unidos (argumentando que Bolivia no cumplía con los objetivos prometidos en el enfrentamiento contra el narcotráfico) implicó el despido de cerca de nueve mil trabajadores ("Página Siete", La Paz, octubre 28 de 2014$)^{5}$. 
De esta manera, a pesar de los discursos a favor de la industrialización, en ambas gestiones se avanzó muy poco en una agenda posextractivista de mediano plazo. Así, el modelo vigente se alimenta sobre la captación de la renta proveniente de las exportaciones y su posterior transformación en ayudas sociales como el bono escolar "Juancito Pinto" y la "Renta Dignidad" para los ancianos.

\section{LA SITUACIÓN LABORAL}

Este panorama económico tiene su correlato en el ámbito de la ocupación. Al igual que en otros países de la región, los datos sobre el nivel de desempleo difieren según si las cifras son emitidas por oficinas estatales o son suministrados por sectores privados. De esta manera, por ejemplo para el año 2011, de acuerdo con datos del órgano oficial (el INE), la tasa de desempleo fue de 5,5\%; mientras que para el CEDLA fue de 7,9\% (INE, 2011 y CEDLA, 2012a). En cuanto al índice de subempleo, de acuerdo con el CEDLA, presentó un incremento del $17 \%$ en 2011 con relación al año 2010 (CEDLA, 2011 y 2012b).

A esta situación hay que sumar que, por un lado, el CEDLA afirma que más del $60 \%$ de la población que figura en las estadísticas como empleada, debe considerarse como autoempleada, debido a que se desempeña en actividades económicas informales. La mayor parte de las fuentes de trabajo posee un escaso nivel de productividad, tanto en el área de comercio, los servicios, como en las faenas agropecuarias de subsistencia donde se abonan los salarios más bajos. Asimismo, a partir de 2011, el trabajo por cuenta propia (sobre todo, en la construcción, los servicios personales y el transporte público) permitió el aumento de la ocupación. Sin embargo, no se informa que es muy seria la precariedad laboral en esas ramas. En ese sentido, el porcentaje de trabajadores que tienen una ocupación estable, bien remunerada y protegida por la seguridad social se redujo en la última década a sólo 20,9\%; el resto tiene un empleo precario (79,1\%) (CEDLA, 2013).

Cabe indicar que los empleos precarios carecen de convenio laboral a la vez que no poseen ni jubilación ni indemnización por 
despido. Este panorama se puede precisar si se considera el acceso a los derechos de salud; así, según el Censo Nacional de Población y Viviendas de 2012, sólo 3.006.226 bolivianos sobre un total de 10.059.856 tienen acceso a un seguro de salud, dato que certifica la precariedad generalizada en el empleo. En resumen, cuando el gobierno hace referencia a la generación de nuevas ocupaciones omite decir que en numerosas ocasiones tuvieron lugar a expensas de su calidad. En ese sentido, se han creado puestos de trabajo vinculados al tipo de desarrollo primario exportador; o sea, no fueron plazas radicadas en las industrias sino en las áreas de trabajadores de servicios y comercio.

De este modo, en el mundo del trabajo, se perpetúa la flexibilización en materia laboral en prejuicio de la calidad del empleo. Sobre todo en lo que refiere a la precarización en los contratos de empleo, se transforma en una herramienta de primer orden para incidir en el deterioro constante de los haberes percibidos. A su vez, esta situación se extiende en forma masiva en el trabajo infantil. Para el año 2015, según el Programa de las Naciones Unidas para el Desarrollo (PNUD), uno de cada cuatro menores de cinco a catorce años desarrolla labores ("Página Siete", La Paz, diciembre 15 de 2015). Por su parte, otros investigadores estiman que más de setecientos niños y jóvenes realizan tareas (desde recolección de desmontes y concentración de minerales hasta preparación de la dinamita) en el Cerro Rico de Potosí (Francescone y Díaz, 2014).

Junto con la flexibilidad en el empleo, los bajos salarios siguen siendo una de las principales variables que caracteriza a este modelo que favorece al gran empresariado convirtiéndose en un especial atractivo para la inversión extranjera en la producción primaria. Por otra parte, la industria manufacturera no le va a la zaga; en general, su competitividad radica en el aumento de la intensidad del trabajo y la suba del volumen de producción sobre la base de la reducción del empleo de mano de obra; en ese sentido, no pocos empleadores tienden a la subcontratación y externalización de diversos procesos o servicios auxiliares, disminuyendo el personal en los establecimientos. 
En cuanto a las medidas de aumento salarial, cabe observar que, en gran parte durante todos estos años, el Poder Ejecutivo adoptó un criterio fiscalista a la hora de decidir el porcentaje de incremento en los haberes. Por lo general, el monto en que se calcula la suba del mismo estuvo sujeta a la tasa inflacionaria del año anterior. Por otro lado, en los últimos años, la pauta salarial solo fue negociada con la $\mathrm{COB}$, dejando a un costado a las cámaras empresariales.

En otro orden de temas, existe una amplia brecha social, ya que la riqueza se concentra en pocas manos: el $20 \%$ de la población más rica acapara más del $60 \%$ de los ingresos, en tanto que el $20 \%$ más pobre sólo recibe el $2 \%$. Según un informe del PNUD, se observa que siete de cada diez bolivianos son indígenas y están insertos en el sector familiar del mercado laboral, con escasas remuneraciones. Por el contrario, en el estrato de mayores ingresos, seis de cada diez no son indígenas y se insertan en el sector empresarial con sueldos relativamente altos y con mejores condiciones ocupacionales (PNUD, 2010). $\mathrm{Al}$ igual de lo que sucede en materia de empleo, las mujeres perciben menos salarios e ingresos que los hombres, por idénticas tareas.

El patrón de desarrollo vigente siguió privilegiando al capital por encima de la fuerza de trabajo. A pesar de las nacionalizaciones de los hidrocarburos y de algunos sectores mineros, el poder real lo siguieron detentando las empresas transnacionales, aportando al fisco porcentajes insignificantes en relación con sus ganancias. De igual modo, el gobierno aumentó sus reservas financieras, manteniendo las mismas en la banca privada y en bonos del tesoro de los países centrales. La pobreza continuó estando presente más allá del incremento de las reservas internacionales en las arcas del Estado y del crecimiento económico. Aunque los líderes del MAS incorporaron en sus discursos la filosofía del "vivir bien", esto no se tradujo en proyectos orientados a la transformación de la matriz productiva del país ni en la disminución de la brecha social. Como se observa en diversos informes publicados por los organismos antes mencionados, la mayor parte de los conflictos desarrollados se originaron por temas económicos: incremento del costo de vida (en particular, en alimentos y transporte), escasez de productos básicos, pugnas por el control de recursos naturales, entre otros. 


\section{EL MUNDO DEL TRABAJO EN LAS COOPERATIVAS MINERAS}

Como hemos explicado la extracción de bienes minerales no renovables sigue teniendo un peso central en la estructura económica del país. A pesar de que en la campańa electoral de 2005 Morales hizo promesas alrededor del renacimiento de la minería estatal bajo la COMIBOL, sus administraciones se caracterizaron por fortalecer al ámbito privado representado tanto por la Federación Nacional de Cooperativas Mineras (FENCOMIN) como por la mediana y gran minería en manos de las transnacionales. Lejos de la renacionalización del subsuelo, el principal interés del gobierno fue de continuar con la estrategia capitalista neoliberal de fomento a los contratos de riesgo compartido entre cooperativistas y empresas extranjeras.

En ese sentido, las presidencias del MAS privilegiaron la relación con el sector cooperativista dentro del mundo de la minería. Desde el inicio de su gobierno, Morales les dio un lugar destacado en su alianza política con el objetivo de atraer a este sector porque, al mismo tiempo que representa un voluminoso número de eventuales votantes, se ha convertido también en el gran creador de empleo para los desempleados provenientes de otros ámbitos laborales. De este modo, el primer ministro que designó para esta área fue un ex presidente de la FENCOMIN. Además, creó el cargo de Viceministro de Cooperativas Mineras, en manos de hombres que previamente habían sido autoridades del área.

Asimismo los cooperativistas actuaron como asambleístas durante la Convención Constituyente y, en la actualidad, también se desempeñan como Diputados, Senadores y representantes diplomáticos.

La política favorable hacia los cooperativistas se evidencia no sólo observando las nuevas áreas que adquirieron bajo su gobierno (muchas de ellas alcanzadas tras cortes de rutas, avasallamientos de yacimientos y movilizaciones) sino también en la obtención de ventajas en términos impositivos y en cambios jurídicos que los beneficia frente a otros actores. Sin embargo, hubo un hecho más importante que expresa la solidez de esta alianza: los cooperativistas consiguieron que la administración del MAS no acceda a la principal demanda de los asalariados mineros, como es la nacionalización 
de los yacimientos y su control por medio de la COMIBOL. Cabe subrayar que todo esto se dio en un escenario signado por la suba de las exportaciones de los bienes del subsuelo, en algunos casos, con la exclusiva participación de los empresarios cooperativistas ${ }^{6}$.

Es útil subrayar que a diferencia de lo que se puede pensar, el cooperativismo minero en Bolivia no es un sistema solidario. Por el contrario, es un sector capitalista que se desarrolla sobre la base de la explotación de sus trabajadores asalariados. Las cooperativas son empresas privadas que buscan denodadamente aumentar su tasa de ganancia sobre la base de un elevado nivel de expoliación laboral de la fuerza de trabajo que contratan.

El sector cooperativista, internamente, no conforma un grupo homogéneo: algunos son empresarios y otros son trabajadores. Detrás del concepto de cooperativas mineras se oculta una compleja realidad en donde se despliega un amplio abanico de situaciones que abarca desde empresas con cerca de treinta miembros hasta otras que emplean a miles de trabajadores. En general, los dirigentes de cooperativas que controlan a las federaciones locales y departamentales se componen de un grupo minoritario de socios que se halla al frente de los órganos de dirección de las cooperativas. Por otra parte, no todos pueden convertirse en socios de una cooperativa minera: para acceder a esa posición se necesita comprar una cuota de admisión que oscila entre los 1.000 y 3.000 dólares.

Cada socio explota una parcela contratando a una cuadrilla de trabajadores mineros donde organiza libremente su producción usufructuando un paraje determinado de un yacimiento. De este modo, se conforma una verdadera unidad productiva, donde se trabaja para el beneficio de unos pocos, con una limitada (o casi nula) capacidad de inversión.

En el seno de las cooperativas se emplean a trabajadores con diversos convenios laborales. Si bien imperan los contratos por medio de salarios preestablecidos, también es normal que se pague según la producción obtenida, sin ningún compromiso salarial previo. Como el nivel de producción en cada cooperativa tiene un alto grado aleatorio, se producen fuertes diferencias en su interior: mientras 
algunos miembros no logran beneficios en varios meses, otros pueden obtener ganancias en forma inmediata. Además de poseer un magro salario, los mineros tienen un empleo inestable. Este fluctúa de acuerdo con los vaivenes en la cotización de los metales en los mercados. La jornada de trabajo, que puede alcanzar hasta dieciséis horas diarias, se efectúa en condiciones altamente precarias: se desarrolla en hábitats donde impera el calor y el frío extremo. El trabajo al interior de la mina requiere un gran esfuerzo físico (la perforación se efectúa con maza y cortafierros); asimismo, cuentan con un equipo básico de protección (el guardatojo y las botas de goma). La insuficiencia de herramientas y maquinarias, junto con la escasez de elementos de seguridad, hace que el empleo en las cooperativas cause numerosos accidentes de trabajo. Si bien en su mayoría los trabajadores al interior de las minas son varones adultos, también en ese circuito productivo se desempeñan mujeres, adolescentes y nińos.

Además de las precarias condiciones laborales, la gran mayoría de las cooperativas carecen de ingenios para el procesamiento de minerales: la concentración de los bienes extraídos se realiza en forma manual. A eso se debe añadir la utilización de productos químicos contaminantes -como el xantato, el ácido sulfúrico, el mercurio- que provocan cuantiosos daños en la salud de los trabajadores. Un detalle no menor es que tampoco las cooperativas efectúan tareas de prospección. La mayoría no planifican la labor en los yacimientos lo que conduce a una disputa permanente y desorganizada por la extracción de las mejores vetas. Este sistema conlleva a que se generen mayores riesgos de accidentes laborales por la falta de conocimiento de las propias condiciones de las zonas productoras que, por lo general, son propensas a inundaciones y derrumbes. Por otro lado, por la forma en que se lleva a cabo la extracción, no están disponibles los recursos para paliar los dańos ambientales que se generan.

En el sistema de explotación que implementan las cooperativas mineras el Estado prácticamente se encuentra ausente en diversos aspectos: desde su nula intervención en las tareas de planificación, prospección y control de las condiciones laborales, hasta en el magro ingreso que recibe vía aporte impositivo a través del Impuesto 
Complementario a la Minería. En idéntico sentido, los socios de las cooperativas comercializan lo extraído con especuladores y rescatadores de minerales evadiendo las contribuciones fiscales.

Conflictos obreros MiNeros DURANTE LA PRIMERA PRESIDENCIA Si bien al comienzo de su primera gestión los principales reclamos que tuvo que hacer frente Morales fueron los que se originaron en el magisterio, los más significativos y neurálgicos fueron los que se produjeron en el mundo minero. De todos estos conflictos, el más importante fue el protagonizado por los trabajadores del subsuelo en Huanuni.

La ciudad de Huanuni, declarada capital del estaño de Bolivia, se encuentra en la primera sección municipal de la provincia Pantaleón Dalence del departamento de Oruro. En dicha zona se alza el yacimiento minero en el cerro Posokoni, que hasta la Revolución de 1952 había pertenecido a uno de los hombres más poderosos de la "rosca": el magnate Simón Patińo. Por otro lado, también el centro productor ha pasado a la historia porque en ese lugar, el 11 de junio de 1944 fue fundado en un congreso de trabajadores mineros la FSTMB. Además, fue el lugar donde se impulsó la realización de un encuentro minero donde se votó la ayuda económica para la guerrilla del Ernesto Che Guevara en 1967.

Tras la debacle provocada por la aplicación del DS 21060, numerosos trabajadores desocupados se instalaron en el cerro Posokoni con el fin de explotar algunas áreas en la zona. A partir de ahí, durante cerca de quince años se produjeron una serie de incidentes entre diversos sectores. Por un lado, los mineros asalariados permanecieron bregando para que el yacimiento siga estando explotado por la COMIBOL; por otra parte, un grupo de empresas transnacionales abogó (y lo logró en forma momentánea) por su privatización y, finalmente, hubo un sector que terminó formando empresas cooperativas para su propio beneficio.

En el año 2006, en el marco de las alianzas establecidas para alcanzar la presidencia, Morales designó como ministro de Minería a Walter Villarroel, miembro de la cooperativa La Salvadora de Huanuni, decisión que generó un abierto malestar con la FSTMB. 
A partir de entonces, los empresarios cooperativistas retomaron la ofensiva para explotar los niveles más profundos del cerro, donde se encontraban trabajando los asalariados de la COMIBOL. En esa coyuntura, en los primeros nueve meses del ańo hubo dieciséis reuniones entre el gobierno y los actores antes mencionados para solucionar la explotación del yacimiento; sin embargo, esto no sirvió para detener los enfrentamientos sangrientos que se sucedieron.

A medida que se desarrollaron las negociaciones, en el mes de septiembre, los cooperativistas agrupados en la FENCOMIN efectuaron una serie de ocupaciones en las minas de Colquiri y de Viloco (ambas del departamento de La Paz) a la vez que bloquearon las principales carreteras del occidente del país exigiendo -entre otras cuestiones- mayores niveles de explotación en Huanuni.

A pesar de que muchas de las demandas solicitadas se cumplieron, en la primera semana de octubre, los cooperativistas incrementaron las presiones sobre la FSTMB y las autoridades nacionales para que se acepten sus reclamos. En ese marco, los socios de las cooperativas que operaban en el cerro determinaron la toma del yacimiento, dando lugar a un enfrentamiento sangriento con los asalariados. Como consecuencia, tras dos días de duros combates, fallecieron dieciséis pobladores y cerca de un centenar de heridos .

Estos hechos derivaron en una grave crisis institucional; en opinión de Morales, este fue uno de los momentos más difíciles de su presidencia. En lo inmediato, Evo solicitó la renuncia del Ministro Villarroel y del director de la COMIBOL, Antonio Rebollo, quienes fueron reemplazados por Guillermo Dalence y Hugo Miranda, respectivamente. A fines de ese mes, el primer mandatario firmó el DS 28901 por el cual se nacionalizó el yacimiento. Además, la empresa estatizada contrató a los empleados mineros que estaban agrupados en las cuatro cooperativas que operaban el cerro; así, ingresaron como asalariados cerca de cuatro mil trabajadores provenientes de las ex cooperativas y de campesinos de la zona (Mokrani, 2006; Fornillo, 2009).

Las protestas de cooperativistas, mineros sindicalizados y empresarios continuaron durante los restantes años de la primera presidencia. Dentro de estas, una de las más importantes fue la que se dio 
desde fines de diciembre de 2008, y a lo largo del primer semestre de 2009, en la empresa Sinchi Wayra, filial de la multinacional suiza Glencore. Las mismas se sucedieron en diversos centros mineros de los departamentos de Potosí, La Paz y Oruro. Los hechos se iniciaron en ocasión de que la compañía había decido despedir a más de mil doscientos trabajadores a la vez que pensaba reducir el quince por ciento de los haberes para aquellos asalariados que permanecían; junto con ello, había dispuesto la reorganización de labores en dos turnos de doce horas diarias. Frente a esa situación en los distintos centros mineros de Porco, Bolívar y Colquiri los trabajadores decidieron efectuar distintas medidas de fuerza. Así, por ejemplo, en este último campamento los asalariados del subsuelo ocuparon la mina y se hicieron cargo de la producción; cabe subrayar que la acción contó con el amplio respaldo de sus mujeres que colaboraron con el bloqueo al ingreso principal del yacimiento.

Ante el impacto de estos acontecimientos y temiendo que la medida se extienda a otros centros mineros, se rubricaron una primera serie de acuerdos entre la FSTMB, la COB, el gobierno y la companía; por medio de ellos, la firma se comprometía a rever los despidos y la rebaja salarial. Sin embargo, esto no sucedió. La multinacional continuó con su ofensiva lo que llevó a que se iniciaran nuevamente acciones de protestas en el mes de mayo con paros y movilizaciones desde los distritos mineros a La Paz con el fin de frenar las ambiciones de la empresa. Tras una masiva marcha de tres mil mineros a la sede del Poder Ejecutivo, se llegó a un nuevo convenio por el cual aumentó la productividad de los trabajadores manteniendo el régimen horario original de ocho horas. En otros términos, el acuerdo avalado por Morales y la $\mathrm{COB}$ terminó siendo perjudicial para los intereses de los trabajadores.

En ese marco es interesante observar cómo fue cambiando el posicionamiento de la dirigencia sindical frente al gobierno. Al principio de su mandato los líderes mantuvieron una actitud de desconfianza hacia el presidente. La central obrera se encontró con una situación novedosa en su larga historia: la presencia de un dignatario indígena con un fuerte respaldo de las organizaciones campesinas. 
En esa coyuntura, la FSTMB y la COB cuestionaron dos grandes temas. En primer lugar, se criticó que el ministerio de minería estuviese encabezado por un socio cooperativista. En segundo término, se esbozó el temor de que sectores sociales vinculados a Evo buscasen modificar el estatuto de la entidad e impusieran a dirigentes de sindicatos campesinos o a cooperativistas al frente de la misma en el XIV Congreso Orgánico que se iba a efectuar en junio de 2006.

Esto último no sucedió ya que los delegados hicieron prevalecer la tradición minera a la hora de elegir una nueva conducción. De esta forma, el encuentro celebrado en Viacha designó a Pedro Montes en reemplazo de Jaime Solares como Secretario Ejecutivo de la central. A la par de esta designación, el cónclave laboral reafirmó la necesidad de "luchar por las agendas de octubre del 2003 y de mayo - junio de 2005 ” por lo que se enfatizó "la nacionalización sin indemnización de los hidrocarburos, la recuperación de todos los recursos naturales y la abrogación de los decretos y leyes neoliberales" (CEDLA, 2009: 5).

A pesar de estas declaraciones, durante los siguientes años la dirigencia encabezada por Montes se terminó acercando al proyecto de Morales, lo que se expresó en el último semestre del 2008. En ese sentido, la decisión de la COB estuvo enmarcada por una serie de sucesos previos. En primer lugar, esta determinación se aceptó tras una serie de fuertes críticas que recibió la dirigencia cobista por parte un importante sector de mineros de Huanuni tras la muerte de dos trabajadores en una medida de fuerza en el mes de agosto ${ }^{7}$.

En segundo lugar, la aproximación al gobierno se encuadró dentro de los distintos realineamientos de fuerzas que se produjeron en esa coyuntura al calor de los tensos enfrentamientos con los prefectos opositores en el marco de los debates de la Asamblea Nacional Constituyente ${ }^{8}$. Paralelo a ello, la disposición del Secretario Ejecutivo de la entidad laboral se inserta en un contexto donde el presidente había sido ampliamente ratificado en las urnas tras el referéndum realizado el 10 agosto.

Toda esta situación condujo a que la dirigencia de la COB, en septiembre de 2008, se integre a la Coordinadora Nacional por el 
Cambio (CONALCAM), ente que aglutinaba a todos los sectores sociales afines al $\mathrm{MAS}^{9}$. Si bien el pacto fue duramente criticado por las centrales obreras departamentales de Oruro y Tarija, el mismo representó la subordinación de la lucha de los asalariados y de la agenda de octubre de 2003 a la defensa del gobierno y su política de conciliación de clase. A partir de entonces, con muy escasas situaciones excepcionales, se produjo una explícita convivencia entre ambos actores a lo largo del primer mandato de Evo.

LA CONFLICTIVIDAD LABORAL MINERA DURANTE EL SEGUNDO MANDATO PRESIDENCIAL

La mayoría de los observadores sobre el proceso reciente boliviano coinciden en afirmar que el segundo período presidencial de Morales se inició dentro de una coyuntura más favorable que su primer mandato: logró un amplio triunfo electoral, derrotando a los sectores opositores de la derecha regional. El MAS se convirtió en un movimiento político y social hegemónico sin precedentes. Sin embargo, ese escenario no alcanzó para evitar que se produzcan numerosos conflictos (Schneider, 2014).

El objetivo gubernamental de impulsar un modelo de desarrollo industrial en el marco del denominado "Proceso de Cambio" provocó, desde el inicio del segundo mandato de Evo, una serie de problemas y cuestionamientos distintos a los experimentados en el primer período. A diferencia de esa gestión, los principales sectores que impugnaron las directivas del Poder Ejecutivo no provinieron del bloque regional del oriente boliviano, sino desde sus propias bases electorales.

Asimismo, como un elemento necesario para promover este modelo de desarrollo, Morales privilegió una estrecha alianza con las corporaciones antiguamente dominantes, poseedoras de las principales riquezas del país y del pasado poder político. En este sentido, no sorprende la abierta aceptación de sus medidas por los enemigos de antańo, como los grupos empresariales de Santa Cruz y Tarija. Por otro lado, el amplio control alcanzado en los diversos órganos del Estado condujo al MAS a contar con una notable concentración 
de poder en todas las instituciones del régimen, lo que en la práctica se tradujo en una clara posición hegemónica sobre las diferentes organizaciones sindicales y comunitarias.

Todo este panorama quedó claramente plasmado en el incremento de las protestas sociales durante el segundo mandato presidencial; en particular, sobresalieron las encabezadas por la clase trabajadora. Según la Fundación UNIR (2012), en el 2010 se desarrollaron 770 conflictos, lo que constituye más del doble de las protestas acaecidas el año anterior, cuando hubo 316, y más del triple de las originadas en 2008. Estos datos cobran una mayor relevancia si se los compara con 2011, cuando se alcanzó la cifra de 1304 conflictos. Por otra parte, si bien en el 2012 y en el 2013 los eventos tendieron a disminuir, en comparación con el 2011, estos nunca alcanzaron los escasos números de la primera gestión de gobierno.

Cabe indicar que el recrudecimiento de la protesta social por parte de la clase obrera se dio en la mayoría de las ocasiones por fuera de la voluntad de la dirigencia sindical. Este panorama se intensificó al cruzarse con las negociaciones anuales salariales impulsadas por la central obrera; en esa coyuntura, frente a las diversas críticas que recibía la dirección de la $\mathrm{COB}$ por su actitud complaciente con Evo, decidió convocar al primer paro general nacional del período. El mismo mostró un masivo apoyo: en los nueve departamentos se realizaron marchas, bloqueos de vías y se instalaron piquetes de huelga de hambre; incluso en $\mathrm{La} \mathrm{Paz}$, los obreros fabriles intentaron tomar el Ministerio de Trabajo y fueron reprimidos por la policía, dejando a numerosos trabajadores detenidos.

Con ese panorama de fondo, se acrecentó el cuestionamiento a la dirección cobista. Los mineros (corazón y alma de la central obrera) reunidos en el XXXI Congreso de la FSTMB criticaron duramente el incumplimiento de Morales; a su vez se demandó la convocatoria a un nuevo Congreso de la COB para renovar el Comité Ejecutivo Nacional junto con el pedido de que Montes sea sometido a un "proceso por el tribunal de honor de la Federación de Trabajadores Mineros" (“La Prensa”, La Paz, septiembre 12 de 2011). 
En esa coyuntura los mineros participaron de numerosos enfrentamientos contra el gobierno. Si bien los empresarios cooperativistas se perjudicaron con la nacionalización del cerro Posokoni en el 2006, el primer mandatario continuó otorgando numerosos beneficios a este sector. En el mismo sentido, el Poder Ejecutivo continuó con su política laboral de no objetar ni castigar a las cooperativas por las pésimas condiciones de empleo que impera en ese espacio laboral (Espinoza, 2010).

En la segunda presidencia, el sector minero continuó beneficiado por el alza de los precios de las materias primas. En parte, esto explica el aumento de las pugnas entre los asalariados y las cooperativas por el control de los yacimientos. Según la pesquisa efectuada, los mayores enfrentamientos, algunos en forma extremadamente violentos, se sucedieron en la región occidental; sobre todo, en aquellas áreas con mayor concentración de minerales, como en los departamentos de La Paz, Potosí y Oruro. Así, por ejemplo, en el año 2011 se efectuaron varias protestas de empresarios cooperativistas, mineros asalariados y campesinos, por el control y explotación de diversos yacimientos de piedra caliza, estańo, hierro y oro, entre otros minerales (Quiroga et al., 2012).

En el año 2012, además de los conflictos por aumentos salariales sobresalieron una serie de enfrentamientos en el mundo minero. De esta manera, se produjo una dura disputa de trabajadores en Colquiri y en Mallku Khota en favor de la nacionalización del subsuelo. En el primer yacimiento, el problema se inició en el mes de mayo cuando un grupo de cooperativistas avasalló la mina ubicada en el departamento de La Paz. Hasta ese momento la empresa era operada bajo la modalidad de riesgo compartido (con la COMIBOL) por la compañía Sinchi Wayra. A partir de ese entonces, durante cinco meses, se desarrolló una puja incesante entre los dos grandes interesados del área: la FSTMB y la FENCOMIN. En ese lapso, se desataron varias provocaciones con dinamita (que ocasionaron decenas de heridos y un muerto), capturas de rehenes, paros en centros mineros, bloqueos de rutas y movilizaciones a la sede del gobierno. Frente a estos hechos, Morales decidió nacionalizar parcialmente el 
yacimiento, pasando su gestión a la COMIBOL; en forma simultánea, dejó que una de las mejores vetas (la denominada Rosario) fuese en un 50\% explotada por los cooperativistas.

En la segunda ocasión, el altercado se dio en torno a la producción de la mina Mallku Khota en territorio indígena originario del Norte de Potosí. Desde el año 2003, la zona venía siendo regenteada por un consorcio subsidiario de la firma canadiense South American Silver. A partir de ese momento, la empresa mantuvo una política de adquisición de concesiones y de cooptación de dirigentes comunitarios por medio de compensaciones sociales y facilitando fuentes de empleo. Sin embargo, esta situación no fue aceptada por la mayoría de la población; esto condujo a que diferentes ayllus del lugar hiciesen numerosas asambleas y cabildos con el objetivo de expulsar a la compañía y exigir al Poder Ejecutivo la reversión de las concesiones otorgadas (Jiménez \& Campanini, 2012). Con ese panorama de fondo, se iniciaron en el mes de mayo una serie de protestas a raíz de que un grupo de policías ingresó con violencia en la comunidad, invadiendo las viviendas, gasificando a los pobladores e intentando capturar a una serie de dirigentes locales. Esto derivó en cinco meses de ocupaciones con rehenes, movilizaciones a La Paz y choques con las fuerzas de seguridad que dejaron un saldo de un campesino muerto y de varios heridos. Tras esos hechos, Morales firmó un Decreto Supremo donde se nacionalizó la reserva, asignando a la COMIBOL la administración del yacimiento ${ }^{10}$. No obstante, este fue un pacto provisorio. Los asalariados de la FSTMB insistieron en que en la medida dispuesta no se contemplara la participación de los cooperativistas y que, en la directiva sindical de los trabajadores de la empresa estatal, se incluyera la participación de los ayllus, ingresando los comunarios de la zona de acuerdo con las necesidades laborales de la COMIBOL. De esta forma, hasta mediados de octubre prosiguieron las pugnas violentas con heridos entre comunarios del lugar y ex cooperativistas y campesinos que apoyaban la presencia de la multinacional canadiense; esto condujo a que Evo decidiera otorgar una custodia militar permanente a la reserva. 
Por fuera de esos problemas específicos, al igual que en Huanuni durante el 2006, en lo anteriormente expuesto se expresa la política del MAS para el sector minero. Por un lado, los trabajadores asalariados exigieron en todo momento la nacionalización de los yacimientos y su control por medio de la COMIBOL; por el otro, los cooperativistas (junto con el gobierno) postularon el derecho del capital privado a la explotación minera. De esta manera, como consecuencia de la radicalización de la propia lucha en torno a la contienda de Colquiri, cuando los cooperativistas bloquearon durante cuarenta y ocho horas todos los caminos del país, la FENCOMIN logró la firma de un Decreto Supremo suscripto por el presidente que alteró por completo los términos de explotación de la Reserva Fiscal Minera, en detrimento del Estado nacional boliviano ${ }^{11}$.

En cuanto a la central obrera, en enero de 2012 se llevó a cabo en Tarija su XV Congreso Ordinario. Dicho encuentro eligió un nuevo Comité Ejecutivo liderado por el ex minero de Huanuni Juan Carlos Trujillo; el evento reiteró un conjunto de declaraciones propias de la historia de la $\mathrm{COB}$ centradas en torno al sindicalismo revolucionario. A pesar de que en ese momento se criticó la relación que mantuvo la anterior dirección cobista con el MAS, a los pocos meses de iniciada la nueva gestión se reiteraron similares prácticas negociadoras con el gobierno como se evidenció en los magros acuerdos salariales de ese ańo y en el apoyo electoral para las elecciones de $2014^{12}$.

En esa coyuntura, en mayo de 2013 el movimiento obrero mantuvo uno de los principales conflictos del período; éste se originó en torno a la Ley de Pensiones No 065, por la cual el gobierno se aferró a mantener los lineamientos básicos diseńados por el ex presidente Gonzalo Sánchez de Losada. La exigencia de la COB se inició en demanda de una mejora sustancial de la renta recibida junto con la posibilidad de cambiar algunos artículos de la mencionada norma. En función de ello, la central sindical dispuso un paro general que tuvo una amplia repercusión, como pocas veces había ocurrido en el último lustro. Durante las dos semanas que se mantuvieron los enfrentamientos, se produjeron cerca de cuarenta puntos de bloqueos en rutas y calles, movilizaciones masivas en cada uno de los 
departamentos del país, huelgas de docentes, los trabajadores del área de la salud, los mineros y los operarios fabriles. Entre algunas de las acciones efectuadas, se destacó el sitio en torno a la Plaza Murillo, en La Paz, que protagonizaron cuatro mil mineros provenientes de Huanuni.

Ante el desarrollo de estas medidas de fuerza el Poder Ejecutivo declaró ilegal la protesta, dispuso la detención de cerca de cuatrocientos trabajadores, reprimió en la localidad de Parotani a los asalariados fabriles y a los mineros en el punto de bloqueo de Caihuasi. En este último sitio, frente al brutal accionar policial que provocó una decena de obreros heridos de bala y cien detenidos, los trabajadores del subsuelo dinamitaron el puente. $\mathrm{Al}$ igual que en otros conflictos, Morales y su vicepresidente descalificaron la puja gremial acusando a los dirigentes sindicales como "golpistas", a la vez que convocaron a algunos de sus grupos afines como la Confederación Nacional de Mujeres Campesinas Indígenas Originarias de Bolivia "Bartolina Sisa" a movilizarse contra los huelguistas y manifestantes obreros.

Tras dieciséis días de paro y luego de varias reuniones mantenidas con emisarios del gobierno, la dirigencia de la $\mathrm{COB}$ redujo el monto de los haberes originalmente solicitados a la par que acordó un cuarto intermedio de negociación con las autoridades ministeriales. Por otra parte, como consecuencia de la protesta, Evo mandó a procesar a veintidós mineros de Huanuni acusados por la voladura del puente de Caihuasi, impulsó la revisión de las cuentas de la Empresa Minera Huanuni buscando suspender el control obrero colectivo y sugirió la posibilidad de que esta última compañía pudiese convertirse en una cooperativa.

Es evidente, como mencionamos al comienzo de este ensayo, que las cuestiones referentes al mundo minero son centrales en Bolivia. Ya sea por el peso que aún mantiene el proletariado del subsuelo como por la dimensión económica del sector. En ese sentido, estos elementos volvieron a cobrar importancia en los primeros meses de 2014 cuando se produjo una serie de conflictos entre los empresarios cooperativistas y el gobierno durante el proceso de discusión parlamentaria de la Ley de Minería y de Metalurgia. 
Si bien el proyecto presidencial continuó con los lineamientos centrales de la norma sancionada bajo la primera presidencia de Gonzalo Sánchez de Lozada en 1997, el gran capital minero y los cooperativistas buscaron una mayor participación en la explotación del subsuelo a expensa de la empresa estatal (la COMIBOL) y de las comunidades originarias.

Al calor de esas discusiones, como en otras disputas, los empresarios cooperativistas se valieron de sus empleados para efectuar un rotundo bloqueo de caminos en el altiplano.

Como consecuencia se dieron una serie de enfrentamientos con la policía que derivaron en el fallecimiento de dos trabajadores junto con medio centenar de heridos. A raíz de ello, el ministro del área Mario Virreira presentó su renuncia, obligando a los parlamentarios a rediseñar el proyecto original del Poder Ejecutivo. En ese escenario, se renegociaron los artículos que generaron las controversias y se aceptó, a fines del mes de abril, una nueva norma legislativa sobre la materia en cuestión.

De este modo, el nuevo marco jurídico impulsa y fomenta la privatización de la actividad minera a través de las grandes empresas y las cooperativas, relega la presencia minera estatal, no prioriza la industrialización de los recursos ni el resguardo del medio ambiente, a la vez que resta importancia a la consulta de los pueblos indígenas sobre el uso de sus tierras.

\section{Conclusiones}

Si bien existen ciertas mejoras en algunos indicadores sociales, el modelo primario exportador, el desempleo, los bajos salarios y la calidad del empleo se mantuvieron entre los problemas más acuciantes de la sociedad boliviana. A pesar de que Evo Morales, en forma permanente, afirma que se encuentra edificando un "socialismo comunitario", el presente artículo observa como continuó privilegiando el interés del capital extranjero y los equilibrios macroeconómicos, en desmedro de las condiciones de vida de los trabajadores.

En la mayoría de los conflictos analizados se observa que estos se iniciaron sin ningún tipo de instancia de previa conversación. Si 
bien de acuerdo con la lectura de la prensa diaria, se puede advertir la existencia de antecedentes en los reclamos, los sectores afectados optaron por la movilización y el propio enfrentamiento, antes que la búsqueda de un entendimiento con las autoridades. Por otro lado, el desenvolvimiento de este accionar les facilitó a los sectores movilizados el posicionamiento en forma rápida de los reclamos en la agenda política y mediática. En cierta manera este comportamiento no es nuevo, sino que hunde sus raíces en una tradición sindical de larga data en Bolivia. Aunque numerosos conflictos se caracterizaron por poseer inusitados niveles de violencia, en particular, por la represión ejercida desde las autoridades, los sectores sociales que se manifestaron lograron acceder a sus objetivos por la fuerza de su movilización y su capacidad de presión. En cierta forma, el presidente muestra su pragmatismo como antiguo líder sindical, el cual se acomoda frente a los reclamos de sus bases en el marco de una lógica caracterizada por la tensión, la negociación y la integración al Estado. De este modo, se vio obligado (en reiteradas oportunidades) a aceptar alguna demanda pidiendo como prenda de cambio la finalización de las medidas de fuerza.

Asimismo, corresponde observar que por distintas circunstancias la dirigencia de la COB cada vez más se ha ido alejando de su propia historia. En ese sentido, no existen por ahora indicios que demuestren un intento de regresar a su antigua independencia de clase frente a los gobiernos en Bolivia.

Bibliografía

ALLENDE, Santiago y Federico BOIDO (2014) "La Bolivia de Evo Morales. Conflictos, tensiones y ambivalencias durante el primer gobierno del MAS (2006-2009)" en SCHNEIDER, Alejandro (Comp.) América Latina hoy. Integración, procesos politicos y conflictividad en su historia reciente, Buenos Aires, Imago Mundi.

ARZE VARGAS, Carlos (2014) Industrialización en el Proceso de Cambio: La modernización populista del MAS, La Paz, CEDLA. 
ARZE VARGAS, Carlos (2016) Una década de gobierno: ¿Construyendo el Vivir Bien o un capitalismo salvaje?, La Paz, CEDLA.

CEDLA 2009 Alerta Laboral, $\mathrm{N}^{\circ} 59$.

CEDLA 2012a Alerta Laboral, $\mathrm{N}^{\circ} 70$.

CEDLA 2013 Alerta Laboral, $\mathrm{N}^{\circ} 71$.

CEDLA 2016 Alerta Laboral, N 76.

CEDLA 2011 Informe, Junio.

CEDLA 2012 b Informe, Junio.

CORTEZ, Jorge (2011) "Las connotaciones político-institucionales del conflicto por el TIPNIS", Andamios, 3.

ESPINOZA, Jorge (2010) Mineria boliviana. Su realidad, La Paz, Plural. FORNILLO, Bruno (2009) "Proletariado minero, nacionalización económica y el posicionamiento actual de la Central Obrera Boliviana" en Polis, $\mathrm{N}^{\circ} 24$.

FORTÚN, Ilya (2012) TIPNIS: la verdad social oculta detrás de la coyuntura y la conflictividad, Tarija, PNUD.

FRANCESCONE, Kirsten y Vladimir DÍAZ (2014) "Entre socios patrones y peones" Petropress 32.

FUNDACIÓN UNIR. (2011) Análisis de la conflictividad del TIPNIS y potencialidad de paz. Cuadernos de Investigación sobre la conflictividad, 1.

FUNDACIÓN UNIR (2012) La conflictividad en Bolivia. Estado de Situación, La Paz, Fundación UNIR.

INSTITUTO BOLIVIANO DE COMERCIO EXTERIOR (2015) Boletín Electrónico Bisemanal No 456.

INSTITUTO NACIONAL DE ESTADÍSTICAS (2012) Censo Nacional de Población y Viviendas, La Paz.

INSTITUTO NACIONAL DE ESTADÍSTICAS (2011) Estadisticas económicas, La Paz.

INSTITUTO NACIONAL DE ESTADÍSTICAS (2014) Estadisticas económicas, La Paz.

JIMÉNEZ, Georgina \& CAMPANINI, Jorge (2012) Minería, tierra y territorio. Mallku Khota. Cochabamba, CEDIB.

LEAÑO, Eduardo (2012) "Movilidad social y conflictos en Bolivia" en Andamios, Nº 6. 
MINISTERIO DE HIDROCARBUROS Y ENERGIA (2011) Balance energético nacional, La Paz.

MINISTERIO DE PLANIFICACIÓN DEL DESARROLLO (2016) Plan de desarrollo económico y social en el marco del desarrollo integral para vivir bien 2016 - 2020. La Paz.

MOKRANI, Dunia (2006) "Pensar la política en Bolivia desde Huanuni" en Pensamiento de los confines, $\mathrm{N}^{\circ} 19$.

PNUD (2010) Informe sobre Bolivia, Noviembre.

QUIROGA, María Soledad et al. (2012) Perfiles de la conflictividad social en Bolivia (2009 - 2011) Análisis multifactorial y perspectivas, La Paz, Fundación UNIR.

RIVASPLATA CABRERA, Francisco et al. (2015). Inversiones de empresas brasileras en América Latina, Bogotá, Editorial Nomos.

SCHNEIDER, Alejandro (2014) "Dificultades políticas y tensiones sociales durante la segunda presidencia de Evo Morales" en SCHNEIDER, Alejandro (Comp.) América Latina hoy. Integración, procesos politicos y conflictividad en su historia reciente, Buenos Aires, Imago Mundi.

URIOSTE, Miguel (2011) Concentración y extranjerización de la tierra en Bolivia, La Paz, Fundación Tierra.

Prensa

El Deber, Santa Cruz de la Sierra

La Prensa, La Paz

La Razón, La Paz

Página Siete, La Paz

Notas de Página

${ }^{1}$ Los datos fueron extraídos de "La Razón”, La Paz, mayo 7 de 2015, "Página Siete", La Paz, septiembre 14 de 2015 y abril 1 de 2016.

${ }^{2}$ Algunos de estos proyectos tuvieron también el efecto de servir como propaganda gubernamental a favor de la gestión de Morales. Véase, el uso mediático que se ha dado a la construcción del satélite 
Túpac Katari por parte de la empresa china Great Wall Industry Corporation con un costo del proyecto que supera los 290 millones de dólares ("Página Siete", La Paz, noviembre 10 de 2013).

${ }^{3} \mathrm{El}$ área fue creada en 1965 como parque nacional en razón de su amplia diversidad biológica y por su enorme riqueza hídrica. En 1990, como resultado de la Marcha por el Territorio y la Dignidad de los pueblos indígenas de tierras bajas, la zona fue reconocida como territorio ancestral de los pueblos mojeño, yuracaré y chimán. Véase: Cortez, Jorge. (2011) "Las connotaciones político-institucionales del conflicto por el TIPNIS”. Andamios, 3.

${ }^{4}$ El Pacto de Unidad fue una organización creada en mayo de 2007 para apoyar la primera gestión del presidente. En oportunidad de la represión de setiembre de 2011, la CIDOB junto con el Consejo Nacional de Ayllus y Markas del Qullasuyu (CONAMAQ) se retiraron del mismo.

${ }^{5}$ En ese escenario, uno de los puntos más ilustrativos de esta situación fue el fracaso del gobierno en el manejo de la administración de la Empresa Pública Nacional Textil (Enatex). Esta compañía había surgido en junio de 2012 luego de que el Estado se hiciera cargo de la compra del complejo industrial conocido como Ametex, que fuera de propiedad del industrial paceño Marcos Iberkleid, tras el cierre del mercado de Estados Unidos.

${ }^{6}$ Durante 2013 la exportación de minerales de las cooperativas fue tres veces mayor a la del sector estatal. Por otro lado, este último sector no participa de la explotación de valiosos minerales como plomo, antimonio y wólfram. "Página Siete", La Paz, abril 13 de 2014.

${ }^{7}$ En esa oportunidad fallecieron dos mineros asalariados de Huanuni como consecuencia del enfrentamiento contra las fuerzas de seguridad del gobierno cuando buscaron levantar el bloqueo de caminos. Este hecho generó una fuerte crisis en el seno del sindicato minero lo que derivó en la renuncia de algunos de sus dirigentes.

${ }^{8}$ Cabe recordar que esa coyuntura estuvo enmarcada por un momento de alta tensión cuando el día 11 de septiembre se produjo la masacre de más de veinte campesinos en Pando ordenada desde la prefectura local. Véase Allende y Boido (2014). 
${ }^{9} \mathrm{El}$ acuerdo fue suscrito en instalaciones de la máxima entidad con la presencia de Morales; el ministro de Trabajo, Walter Delgadillo; el presidente interino de Yacimientos Petrolíferos Fiscales Bolivianos, Santos Ramírez, el titular del CONALCAM, Fidel Surco; el ejecutivo de la Central Obrera Regional de El Alto, Edgar Patana, entre otros.

${ }^{10}$ Luego de suscribir el acuerdo, el primer mandatario reconoció su "error" por "no convocar" en forma inmediata a los comunarios de la región para resolver el problema, y por ello, ofreció sus disculpas. "Página Siete", La Paz, julio 11 de 2012.

${ }^{11}$ Hasta ese entonces, el Estado boliviano era el único propietario de los recursos mineralógicos de la Reserva Fiscal Minera. Esta norma legal, desde sus orígenes, había sido duramente cuestionada por los cooperativistas y los empresarios privados, nacionales y extranjeros. Con la nueva disposición legal, Morales acalló estas críticas.

${ }^{12}$ Esta negociación se dio a pesar de las numerosas protestas que se dieron a favor de un importante incremento salarial. Información extraída de "La Razón”, "La Prensa”, "Página Siete", La Paz, mayo de 2012.

${ }^{13}$ Una crónica detallada en "La Razón”, "La Prensa” y "Página Siete”, La Paz, mayo a septiembre de 2013.

Fecha de Recepción del Artículo: 3 de abril de 2016 Fecha de Aprobación: 3 de junio de 2016 